Draft version August 12, 2021

Preprint typeset using $\mathrm{LATEX}_{\mathrm{E}}$ style emulateapj v. 5/2/11

\title{
ALIGNMENTS OF GALAXIES WITHIN COSMIC FILAMENTS FROM SDSS DR7
}

\author{
Youcai Zhang ${ }^{1}$, XiaOhu Yang ${ }^{1,2}$, Huiyuan Wang ${ }^{3,4}$, Lei Wang ${ }^{5}$, H. J. Mo ${ }^{4}$, Frank C. van Den Bosch ${ }^{6}$ \\ Draft version August 12, 2021
}

\begin{abstract}
Using a sample of galaxy groups selected from the Sloan Digital Sky Survey Data Release 7 (SDSS DR7), we examine the alignment between the orientation of galaxies and their surrounding large scale structure in the context of the cosmic web. The latter is quantified using the large-scale tidal field, reconstructed from the data using galaxy groups above a certain mass threshold. We find that the major axes of galaxies in filaments tend to be preferentially aligned with the directions of the filaments, while galaxies in sheets have their major axes preferentially aligned parallel to the plane of the sheets. The strength of this alignment signal is strongest for red, central galaxies, and in good agreement with that of dark matter halos in N-body simulations. This suggests that red, central galaxies are well aligned with their host halos, in quantitative agreement with previous studies based on the spatial distribution of satellite galaxies. There is a luminosity and mass dependence that brighter and more massive galaxies in filaments and sheets have stronger alignment signals. We also find that the orientation of galaxies is aligned with the eigenvector associated with the smallest eigenvalue of the tidal tensor. These observational results indicate that galaxy formation is affected by large-scale environments, and strongly suggests that galaxies are aligned with each other over scales comparable to those of sheets and filaments in the cosmic web.
\end{abstract}

Subject headings: large-scale structure of universe - methods: statistical - cosmology: observations

\section{INTRODUCTION}

Numerical simulations and large galaxy redshift surveys have revealed a striking cosmic web, composed of clusters, filaments, sheets and voids as the primary building blocks of the large scale structure (see Bond et al. 1996, and references therein). According to the standard paradigm for galaxy formation, these structure arise from gravitational instability amplifying primordial, gaussian density fluctuations in a nearly homogeneous and isotropic early Universe. Galaxies are the visible component of gravitationally bound systems dominated by dark matter halos, which form via gravitional collapse of density perturbations that become nonlinear (e.g., Mo et al. 2010). Since dark matter halos grow in mass by accretion, and are subjected to the tidal forces from their surroundings, the structure and formation of both dark matter halos and galaxies is affected by their large-scale environment (e.g., White 1984; Bvrd \& Valtonen 1990; Gottlöber et al. 2001; Aragon-Calvo 2012; Muldrew et al.|2012).

From a dynamical point of view, matter tends to flow out of the voids, then accretes onto the sheets, collapses to the filaments, and finally accumulates onto large clusters at the intersection of multiple

\footnotetext{
${ }^{1}$ Key Laboratory for Research in Galaxies and Cosmology, Shanghai Astronomical Observatory; Nandan Road 80, Shanghai 200030, China; E-mail: yczhang@shao.ac.cn

${ }^{2}$ Center for Astronomy and Astrophysics, Shanghai Jiao Tong University, Shanghai 200240, China; Email: xyang@sjtu.edu.cn

${ }^{3}$ Key Laboratory for Research in Galaxies and Cosmology, University of Science and Technology of China, Hefei, Anhui 230026, China

${ }^{4}$ Department of Astronomy, University of Massachusetts, Amherst MA 01003-9305, USA

${ }^{5}$ Purple Mountain Observatory, the Partner Group of MPI für Astronomie, 2 West Beijing Road, Nanjing 210008, China

${ }^{6}$ Department of Astronomy, Yale University, P.O. Box 208101 New Haven, CT 06520-8101, USA
}

filaments. This accretion history is expected to impact the properties of halos and galaxies formed in them (e.g. Zhao et al. 2003, 2009), as well as their clustering strength (Sheth \& Tormen 2004; Gao et al. 2005; Jing et al. 2007; Wang et al. 2007; Dalal et al. 2008; Hahn et al. 2009). Indeed, numerous studies have found, using numerical simulations, that the spins and/or shapes of dark matter halos are aligned with their large-scale structure (Faltenbacher et al. 2002; Colberg et al. 2005; Kasun \& Evrard 2005; Basilakos et al. 2006; Bett et al. 2007; Cuesta et al. 2008; Paz et al. 2008; Zhang et al. 2009; Wang et al. 2011; Codis et al. 2012; Trowland et al. 2013). In particular, the major axes of halos are found to lie preferentially on the shells of voids (Patiri et al. 2006; Brunino et al. 2007; Cuesta et al. 2008), in the plane of sheets (Aragón-Calvo et al. 2007; Hahn et al. 2007; Zhang et al. 2009), and along the direction of filaments (Altav et al. 2006; Aragón-Calvo et al.|2007; Hahn et al. 2007; Zhang et al. 2009; Libeskind et al. 2013). Since galaxy properties, such as spin and shape, are expected to be affected by their host halos (Berta et al. 2008; Debattista et al. 2013), alignments between galaxies and large-scale environment are also expected in the current paradigm of galaxy formation (Jimenez et al. 2010).

Observationally, numerous studies have characterized various forms of alignment between the orientation of galaxies and their large scale environment. These include the alignment between the orientation of central galaxies and the spatial distribution of their satellite galaxies, or the major axis of its host group (Sales \& Lambas 2004; Brainerd 2005; Yang et al. 2006; Azzaro et al. 2007; Faltenbacher et al. 2007; Wang et al.|2008, 2010; Agustsson \& Brainerd 2010; Nierenberg et al. 2011; Li et al. 2013), the alignment between the orientation of satellite galaxies and central-satellite position vector (Pereira \& Kuhn 2005; Faltenbacher et al. 2007; 
Hao et al. 2011; Schneider et al. 2013), and the alignment between galaxy group shape and the large scale structure (Faltenbacher et al. 2009; Wang et al. 2009; Godłowski et al. 2010; Godłowski 2011, 2012; Paz et al. 2011; Wang et al. 2011; Skielboe et al. 2012; Li et al. 2013). All these observational studies reveal a strong alignment between galaxies and their surrounding largescale structure. Moreover, the strength of the alignment is found to depend on a variety of galaxy properties. Typically, the alignment effects are found to be much stronger for red galaxies than for blue galaxies, and to increase with the mass of the galaxy or its host halo. For example, using a large galaxy redshift catalog from SDSS DR6, Faltenbacher et al. (2009) revealed an overabundance of reference galaxies along the major axes of red galaxies out to projected distances of $60 \mathrm{~h}^{-1} \mathrm{Mpc}$, but no alignment signal was detected for blue galaxies. Using the CMASS galaxy sample at $z \sim 0.6$, Li et al. (2013) found that more massive galaxies are more strongly aligned with the large-scale structure up to scales of $70 h^{-1} \mathrm{Mpc}$. Using galaxies in the SDSS, Tempel et al. (2013b) and Tempel \& Libeskind (2013) found that the spin axis of bright spiral galaxies aligns with the host filament but with significant correlation with the sheet normal.

Theoretically, alignment studies with $N$-body simulations have so far focused on statistics on the orientations of halos with respect to directions defined by cosmic web, such as filaments, sheets or voids (Brunino et al. 2007; Zhang et al. 2009; Wang et al. 2011). The alignment signals found here are in general much stronger than those obtained in observations for galaxies. Such kind of difference indicates that, due to complex accretion and merger histories, there is a misalignment between galaxies and their host halos. Indeed, Faltenbacher et al. (2009) found a mean projected misalignment between a halo and its central region of $\sim 25^{\circ}$. Wang et al. (2008) suggested the misalignment followed a Gaussian distribution with a mean of $0^{\circ}$ and a dispersion of $\sim 23^{\circ}$ (see also Okumura et al. 2009; Li et al. 2013). Using hydrodynamical simulations, Hahn et al. (2010) found that galaxy spin is strongly aligned with the inner dark matter halo regions where the discs reside, but the alignment of galaxy spin with the spin of the entire host halo is much weaker. Nevertheless, massive galaxy discs do have spins preferentially aligned with their host filaments, while the spin of discs in lower mass halos shows alignment with the intermediate principal axis of the large-scale tidal field (Hahn et al. 2010).

In this paper, we study the alignments between galaxy shapes and the surrounding large-scale environments, such as filaments and sheets. Different from earlier investigations, here we use a large galaxy group catalog constructed from the Sloan Digital Sky Survey Data Release 7 (SDSS DR7, Abazajian et al. 2009) so that we can distinguish between central and satellite galaxies. Furthermore, we use the cosmic tidal field in the SDSS DR7 survey volume directly reconstructed from the group catalog, using the method of Wang et al. (2012) that has been shown to be reliable. Based on the signs of the eigenvalues of this tidal field we classify the cosmic web into four categories: clusters, filaments, sheets, and voids. We then measure the angles between the projected orientations of the major axes of galaxies and the eigenvectors of the local tidal tensor, and study how these alignments depend on galaxy properties. In order to interpret our results, we compare our findings with the (projected) alignments of dark matter halos and their cosmic web environment obtained from a large $N$-body simulation.

This paper is organized as follows. In Section 2, we present the data and the methodology used to measure the various alignment signals, both for galaxies in the SDSS DR7 and for dark matter halos in our $N$-body simulation. The alignments of galaxies and dark matter halos with respect to the cosmic web are presented in Section 3. and we summarize and discuss our results in Section 4 .

\section{DATA AND METHODOLOGY}

In this section we outline how we characterize the cosmic web using galaxy groups identified in the SDSS DR7 galaxy catalog, and we describe the method used to measure the alignment between galaxies and structures, such as filaments and sheets, identified in this cosmic web. We also describe a similar methodology applied to numerical $N$-body simulations, which we use to interpret our findings.

\subsection{Observational Data}

The SDSS (York et al. 2000), one of the most influential galaxy redshift surveys, is a multi-band imaging and spectroscopic survey to explore the largescale distribution of galaxies and quasars. The galaxy sample used here is constructed from the New York University Value-Added Galaxy Catalog (NYU-VAGC; Blanton et al. 2005), which is based on the SDSS Data Release 7 (Abazajian et al. 2009), but with an independent set of significantly improved reductions over the original pipeline. Marking the completion of the survey phase known as SDSS-II, DR7 features spectroscopy that is now complete over a large contiguous area of the Northern Galactic Cap, closing the gap which was present in previous data releases. The continuity over this large area is a great advancement and critical to the statistics of large-scale structure. From the NYU-VAGC, we select all galaxies in the Main Galaxy Sample with an extinction-corrected apparent magnitude brighter than $r=17.72$, with redshifts in the range $0.01 \leq z \leq 0.20$ and with redshift completeness $\mathcal{C}_{z}>0.7$. The extracted SDSS galaxy catalog contains a total number of 639,555 galaxies with a sky coverage of $\sim 7,750$ square degrees. We have complemented this sample with a small subset of galaxies that do not have SDSS spectra, but for which we have used the redshifts kindly made available to us from the Korea Institute from Advanced Study (KIAS) ValueAdded Galaxy Catalog (Park \& Choi 2005; Choi et al. 2007; Choi et al. 2010).

Galaxy groups are selected using the adaptive halobased group finder developed by Yang et al. (2005, 2007). First potential group centers are identified using the standard FOF algorithm or an isolation criterion. Next, the total group luminosity, defined as the summed luminosity of all group members, is converted into an estimate for the group mass using an assumed mass-to-light ratio. This mass estimate is used to compute the expected velocity dispersion and angular size of the corresponding dark matter halo, which in turn are used to select group 


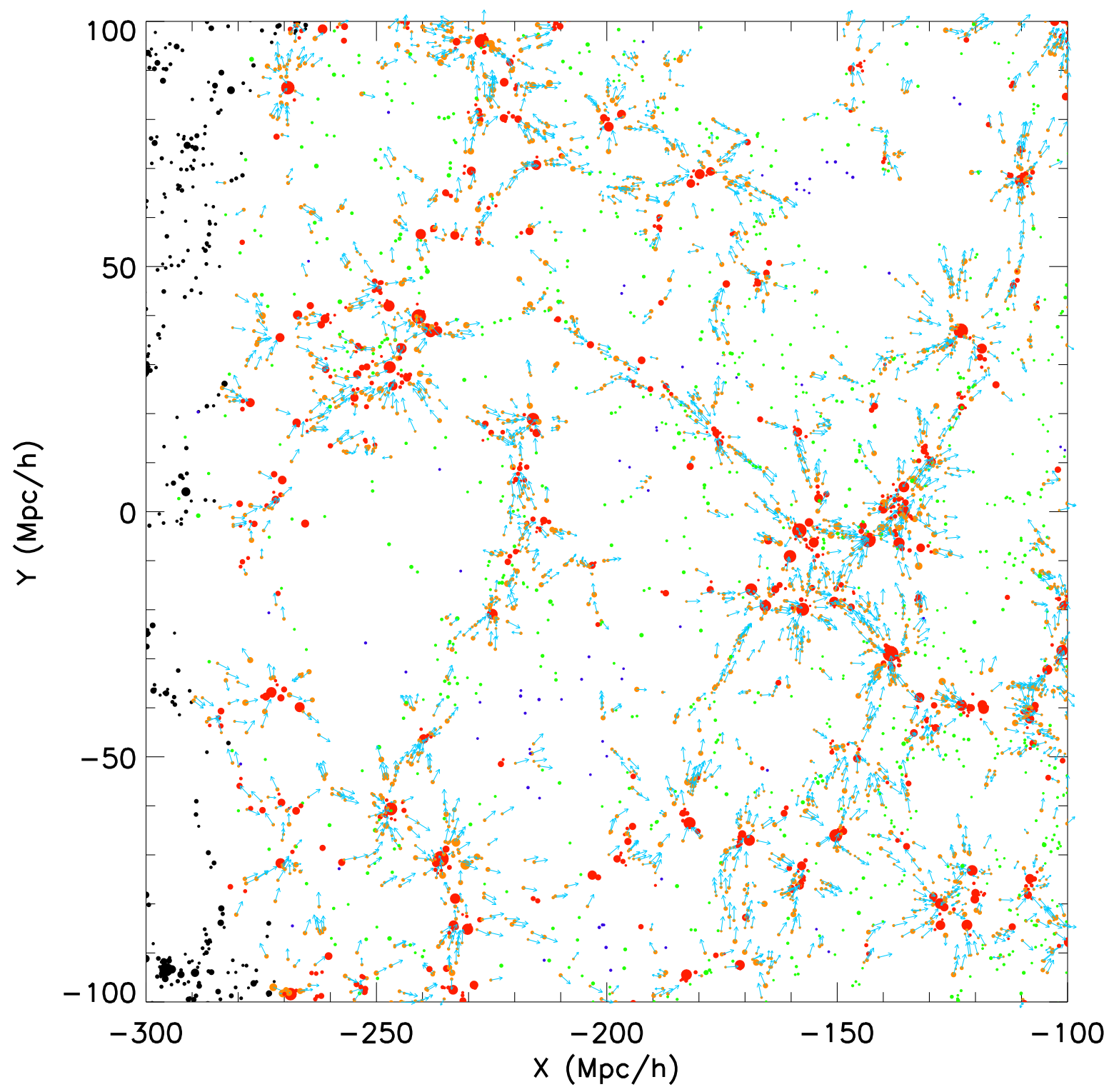

FIG. 1. - Galaxy distributions and environmental classifications in a slice of thickness $10 h^{-1} \mathrm{Mpc}$ from SDSS DR7. The galaxy groups in four different environments are classified by different colors: clusters (red), filaments (orange), sheets (green), voids (blue). The cyan arrow indicates the direction of the filament at the center of each group. Black dots are groups with $z>0.12$ and therefore fall outside our survey volume, i.e., that have a filling factor $F=0$ (see text), and are therefore not included in the analysis..

members in redshift space. This method is iterated until group memberships converge (see Yang et al. 2007, for details).

Applying our group finder to the sample of 639,555 galaxies described above yields a catalog of 472,532 galaxy groups (including systems with single galaxies). Since the group luminosities are strongly correlated with halo masses, we assign halo masses to our groups based on the ranking of the total, characteristic group luminosity, defined as the summed luminosity of all group members with $r$-band absolute magnitude ${ }^{0.1} M_{r}-5 \log h \leq$ -19.5 . Here the absolute magnitudes of galaxies are all $K$-corrected and evolution corrected to $z=0.1$, using the method described in Blanton et al. (2003). The resulting mass assignment is complete to $z \sim 0.12$ for groups with mass $M_{\mathrm{h}} \gtrsim 10^{12} h^{-1} \mathrm{M}_{\odot}$. Hence, in what follows we restrict our analysis to the nearby volume covering the redshift range $0.01 \leq z \leq 0.12$, and within the large continuous region covering the north galactic cap. Using all galaxy groups with an assigned mass $M_{\mathrm{h}} \geq$ $M_{\mathrm{th}}=10^{12} h^{-1} \mathrm{M}_{\odot}$, Wang et al. (2012) constructed the mass, tidal and velocity (SDSS-MTV) fields for this volume. In what follows, we will use these fields to quantify the cosmic web. In order to account for boundary effect that might impact the SDSS-MTV field construction, Wang et al. (2012) introduced the 'filling factor' parameter, $F$, which characterizes the distance of a galaxy group to the survey boundary. For each group inside the volume covering the redshift range $0.01 \leq z \leq 0.12$, the filling factor $F$ is defined as the fraction of the survey 
volume in a sphere of radius $R_{F}$ centered on this group. Generally, large $F$ means that the group is located in the inner region of the survey volume, while small $F$ means the group is near the boundary. Throughout we adopt $R_{F}=80 h^{-1} \mathrm{Mpc}$, and we have tested that our results are insensitive to changes in $R_{F}$ of a factor of two. We also emphasize that we have found the alignment signals to be insensitive to how we cull our sample based on $F$; using only groups with $F>0.9$ or all groups with $F>0$ results in differences in the average misalignment angles (see below for definition) of $<0.1^{\circ}$. Hence, in what follow we use all groups with $F>0$.

\subsection{Characterizing the Cosmic Web}

In specifying the SDSS-MTV fields, Wang et al. (2012) use the tidal tensor field

$$
T_{i j}(\boldsymbol{x})=\frac{\partial^{2} \phi}{\partial x_{i} \partial x_{j}},
$$

where $i$ and $j$ are indices with values of 1,2 , or 3 , and $\phi$ is the peculiar gravitational potential, which can be calculated from the distribution of dark matter halos with mass $M_{\mathrm{h}}$ above some threshold value $M_{\mathrm{th}}$ through the Poisson equation,

$$
\nabla^{2} \phi=4 \pi G \bar{\rho} \delta=4 \pi G \bar{\rho} \delta_{\mathrm{h}} / b_{\mathrm{h}} .
$$

Here $\bar{\rho}$ is the average density of Universe, $\delta(\boldsymbol{x})=$ $\rho(\boldsymbol{x}) / \bar{\rho}-1$ is the matter overdensity field, $\delta_{\mathrm{h}}(\boldsymbol{x})$ is the overdensity field of dark matter halos (groups) with mass $M_{\mathrm{h}} \geq M_{\mathrm{th}}$, whose average, linear bias parameter is given by $b_{\mathrm{h}}$. Thus, at the location of each group we can derive $\phi$ and $T_{i j}$ using the distribution of groups with $M_{\mathrm{h}} \geq M_{\mathrm{th}}$ (see Wang et al. 2012). Briefly, we first transform the redshift and sky coordinates for each group into a Cartesian coordinate system using

$$
\begin{aligned}
X & =R(z) \cos \delta \cos \alpha \\
Y & =R(z) \cos \delta \sin \alpha \\
Z & =R(z) \sin \delta,
\end{aligned}
$$

where $\alpha$ and $\delta$ refer to the right ascension and declination, respectively, and $R(z)$ is the comoving distance out to redshift $z$. Next, the halo overdensity field, $\delta_{\mathrm{h}}$, is computed on a rectangular $(X, Y, Z)$ grid. This field is then corrected for redshift space distortions using the method of Wang et al. (2009), which is based on linear theory. Subsequently, Fast Fourier Transform is used to obtain the potential field, $\phi$, by solving the Poisson equation (2), after which derivative operators are applied (in Fourier space) to derive the tidal tensor.

This tidal tensor is subsequently diagonalized to obtain the eigenvalues $\lambda_{1} \geq \lambda_{2} \geq \lambda_{3}$ of the tidal tensor at the position of the group, which, in analogy with Zel'dovich theory (Zeldovich 1970), can be used to classify the group's environment in one of four classes:

- cluster : a point where all three eigenvalues are positive

- filament : a point where $T_{i j}$ has one negative and two positive eigenvalues

- sheet : a point where $T_{i j}$ has two negative and one positive eigenvalues
- void : a point where all three eigenvalues are negative

(see Hahn et al. 2007a, b). Of the 277,139 galaxy groups with $M_{\mathrm{h}} \geq M_{\mathrm{th}}=10^{12} h^{-1} \mathrm{M}_{\odot}$ in our survey volume, 41,908 groups $(15.1 \%)$ are located in a cluster environment, 173,820 groups (62.7\%) are located in a filament , 57, 169 groups (20.6\%) are located in a sheet, and 4,242 group (1.5\%) are located in a void . Fig. 1 shows the distribution of galaxy groups from the SDSS DR7 in a $200 h^{-1} \mathrm{Mpc} \times 200 h^{-1} \mathrm{Mpc}$ slice of thickness $10 h^{-1} \mathrm{Mpc}$, in which groups in different environment classes are indicated with different colors (see also Wang et al. 2012).

\subsection{Alignment Angles}

The main goal of this study is to characterize to what extent the orientation of galaxies is aligned with the structure of its surrounding cosmic web (i.e., the orientation of filaments and sheets). To that extent we consider two alignment angles: for galaxies whose group is located in a filament environment, we compute the angle $\theta_{\mathrm{GF}}$, defined as the angle between the orientation of the galaxy, $\theta_{\mathrm{G}}$, and the direction of the filament, $\theta_{\mathrm{F}}$. For galaxies whose group is located in a sheet environment, we instead compute the angle $\theta_{\mathrm{GS}}$, defined as the angle between $\theta_{\mathrm{G}}$ and the normal direction of the sheet $\theta_{\mathrm{S}}$.

The orientation angle $\theta_{\mathrm{G}}$ of the major axis of a galaxy, projected on the sky, is specified by the 25 magnitudes per square arcsecond isophote in the r-band. The orientation angle of a filament, $\theta_{\mathrm{F}}$, at the location $\boldsymbol{x}$ of a filament group is computed as follows. First we identify the 3D direction $\Delta \boldsymbol{x}$ of the filament with the eigenvector corresponding to the single negative eigenvalue of the tidal tensor at $\boldsymbol{x}$. Next, we compute the projected direction of the filament on the sky using

$$
\theta_{\mathrm{F}}=\arctan \left[\frac{\Delta \alpha \cos \delta_{\boldsymbol{x}}}{\Delta \delta}\right],
$$

where $\Delta \alpha$ and $\Delta \delta$ are the right ascension and declination differences, respectively, between the locations $\boldsymbol{x}$ and $\boldsymbol{x}+\Delta \boldsymbol{x}$, and $\delta_{\boldsymbol{x}}$ is the declination at $\boldsymbol{x}$. Similarly, the projected orientation $\theta_{\mathrm{S}}$ of the normal vector of a sheet, at the location $\boldsymbol{x}$ of a sheet group, is computed using the same equation (4), but now $\Delta \boldsymbol{x}$ is the 3D eigenvector corresponding to the single positive eigenvalue of the tidal tensor at $\boldsymbol{x}$. As an illustration, the cyan arrows in Fig. 1 indicate the orientations of filaments projected along the Cartesian coordinate $Z$ in equation (2). Note that only groups in a filament environment, have such a cyan arrow . Visual inspection of Fig. 1 reveals that the filament directions agree nicely with the prominent filamentary structures that are visible solely from the spatial distribution of groups in the cosmic web.

In order to quantify possible alignments and their significance, we generate 100 random samples in which the orientations of filaments and sheets $\left(\theta_{\mathrm{F}}\right.$ and $\left.\theta_{\mathrm{S}}\right)$ are kept fixed, but the orientations of the galaxies, $\theta_{\mathrm{G}}$, are randomized. The alignment signal can then be expressed by

$$
P\left(\theta_{\mathrm{GX}}\right)=\frac{N\left(\theta_{\mathrm{GX}}\right)}{\left\langle N_{\mathrm{R}}\left(\theta_{\mathrm{GX}}\right)\right\rangle},
$$

where $X$ either stands for ' $\mathrm{F}$ ' or ' $\mathrm{S}$ ', $N\left(\theta_{\mathrm{GX}}\right)$ is the num- 
ber of galaxy-filament or galaxy-sheet pairs in each bin of alignment angle $\theta_{\mathrm{GX}}$, and $\left\langle N_{\mathrm{R}}\left(\theta_{\mathrm{GX}}\right)\right\rangle$ is the average number of such pairs obtained from the 100 random samples. For the random samples, we also compute the standard deviation of $P_{\mathrm{R}}\left(\theta_{\mathrm{GX}}\right)=N_{\mathrm{R}}\left(\theta_{\mathrm{GX}}\right) /\left\langle N_{\mathrm{R}}\left(\theta_{\mathrm{GX}}\right)\right\rangle$, which is used to assess the significance of any detected alignment signal. Since the significance of any alignment is quantified with respect to the null hypothesis, throughout this paper, we plot the error bars on top of the $P_{\mathrm{R}}\left(\theta_{\mathrm{GX}}\right)=1$ line. In addition to the PDFs, we also calculate the mean angle $\left\langle\theta_{\mathrm{GX}}\right\rangle$ and $\sigma_{\theta_{\mathrm{GX}}}$, which is the the standard deviation of $\left\langle\theta_{\mathrm{GX}}\right\rangle_{\mathrm{R}}$ for the 100 random samples. The angle $\theta_{\mathrm{GX}}$ is constrained in the range $0^{\circ} \leq \theta_{\mathrm{GX}}<90^{\circ}$. In the absence of any alignment, $P\left(\theta_{\mathrm{GX}}\right)=1$ and $\left\langle\theta_{\mathrm{GX}}\right\rangle=45^{\circ}$. Values of $\left\langle\theta_{\mathrm{GX}}\right\rangle<45^{\circ}$ indicate that the galaxy orientations tend to be parallel to the filaments or the normal directions of the sheets, while $\left\langle\theta_{\mathrm{GX}}\right\rangle>45^{\circ}$ indicates perpendicular orientations.

\subsection{Comparison with $N$-body Simulations}

In order to facilitate the interpretation of our analysis, we also use a N-body simulation carried out at the Shanghai Supercomputer Center using the massively parallel TreeSPH GADGET-2 code (Springel 2005). The simulation evolved $1024^{3}$ dark matter particles in a periodic box of size $L=100 h^{-1} \mathrm{Mpc}$. Glass-like cosmological initial conditions (White 1996) were generated at redshift $z=120$ using the Zel'dovich approximation. The particle mass and softening length are $6.93 \times 10^{7} h^{-1} \mathrm{M}_{\odot}$ and $2.25 h^{-1} \mathrm{kpc}$, respectively. The adopted cosmological parameters are $\Omega_{\mathrm{m}}=0.25, \Omega_{\mathrm{b}}=0.045, \Omega_{\Lambda}=0.75$, $h=0.73, n=1$ and $\sigma_{8}=0.9$.

Dark matter halos are identified from the $z=0$ simulation output using the standard FOF algorithm (Davis et al. 1985) with a linking length of 0.2 times the mean inter-particle separation. To obtain a reliable measurement of the halo shape, we only retain halos with at least 500 particles for further analysis, resulting in a catalog with 73,068 halos covering the mass range from $3.5 \times 10^{10} h^{-1} \mathrm{M}_{\odot}$ to $6.8 \times 10^{14} h^{-1} \mathrm{M}_{\odot}$.

The shape of a FOF halo containing $N$ particles is determined using the moment of inertia tensor

$$
I_{\alpha \beta}=m \sum_{i=1}^{N} x_{i, \alpha} x_{i, \beta},
$$

where $m$ is the mass of a dark matter particle, and $x_{i, \alpha}$ denotes the $\alpha$-component of the position vector of particle $i$ relative to the center of mass. The axis ratios of the halo are proportional to the square roots of the corresponding eigenvalues, and the orientation of the halo is determined by the corresponding eigenvectors.

In the simulation, we have extracted the cosmic filaments and sheets using the Density Field Hessian Matrix Method described in Zhang et al. (2009) (see also Hahn et al. 2007b). This method uses the eigenvalues of the Hessian matrix of the smoothed mass density field (smoothed with a Gaussian filter with a smoothing length $R_{\mathrm{s}}=2.1 h^{-1} \mathrm{Mpc}$ ) to classify the cosmic web environment of a dark matter halo, similar to the method used above based on the tidal tensor. The directions of the filaments and sheets are again based on the directions of the eigenvectors as in 2.3. In Zhang et al. (2009),

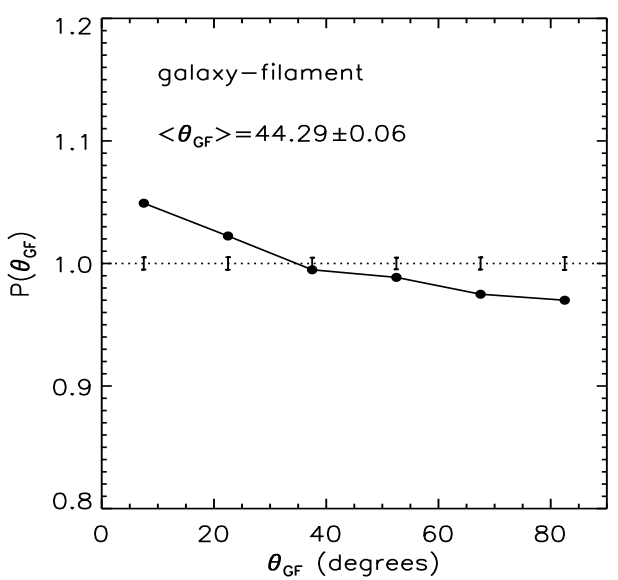

FIG. 2.- Normalized probability distribution of the angle $\theta_{\mathrm{GF}}$ between the projected orientation of the major axis of SDSS galaxies and that of the filament in which its group resides. The horizontal dotted line corresponds to an isotropic distribution of alignment angles, while the error bars indicate the scatter obtained from 100 realizations in which the orientations of the galaxies have been randomized. The average value of $\theta_{\mathrm{GF}}$ and its error (obtained from the 100 random realizations) are indicated.

this simulation and methodology were used to study the alignment between the spins and shapes of dark matter halos and their large scale environment. They find that both the spin and the major axes of $f i l a m e n t$ halos with masses $\lesssim 10^{13} h^{-1} \mathrm{M}_{\odot}$ are preferentially aligned with the direction of the filaments. The spins and major axes of sheet halos were found to lie preferentially parallel to the sheets (see also Altay et al.2006; Aragón-Calvo et al. 2007; Hahn et al. 2007).

Here, in order to compare with the alignment signals detected in the SDSS data, we project the orientations of the halos, filaments and sheets along one of the simulation's coordinate axes. Subsequently, we compute the probability distribution functions $P\left(\theta_{\mathrm{HF}}\right)$ and $P\left(\theta_{\mathrm{HS}}\right)$, where $\theta_{\mathrm{HF}}$ is the angle between the projected orientation of the major axis of a filament halo and that of its filament, while $\theta_{\mathrm{HS}}$ is the angle between the projected orientation of the major axis of a sheet halo and the projected normal axis of the sheet.

\section{RESULTS}

To quantify the impact of filaments and sheets onto galaxies, we investigate the orientations of galaxies relative to the directions of the filaments or the normal vectors of the sheets in which the galaxies reside. For simplicity, we will often use the terms "shape", as well as "filament" and "sheet", as an indication of the direction in the obvious sense.

\subsection{The Alignment between Galaxy Shape and Filament}

Fig. 2 shows the probability distribution $P\left(\theta_{\mathrm{GF}}\right)$ of the angle between the projected major axis of filament galaxies and the projected direction of their filaments, as measured from the SDSS DR7 galaxy catalog using the method described in 2 . With an average alignment angle of $\left\langle\theta_{\mathrm{GF}}\right\rangle=44.29^{\circ} \pm 0.06^{\circ}$, there is a clear, and highly significant, indication that $f i l a m e n t$ galaxies are preferentially aligned parallel to their filaments 


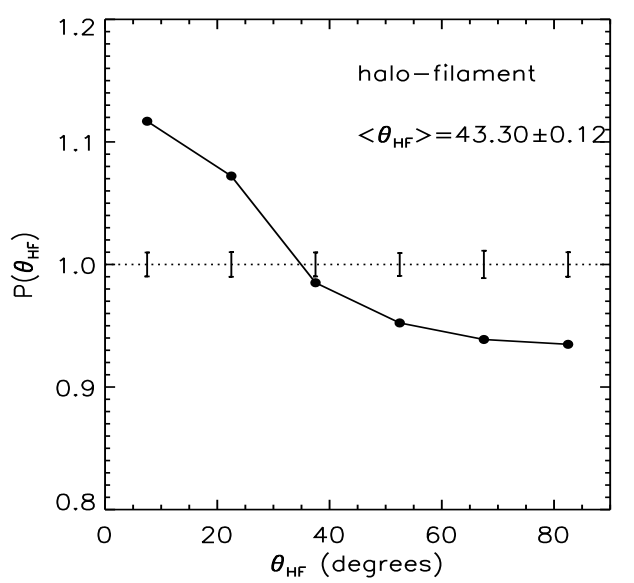

FIG. 3.- Same as Fig.2 but for the alignment angle $\theta_{\mathrm{HF}}$ between the projected orientation of a dark matter halo and that of the filament in which it resides. These results have been obtained using the numerical $N$-body simulation described in 2.4

Fig. 3 shows the equivalent plot for $\theta_{\mathrm{HF}}$, the alignment angle between the projected orientation of filament dark matter halos and that of the filament in which they reside. As in the SDSS data, their is a clear and significant alignment between halos and filaments, in the sense that the major axis of dark matter halos preferentially aligns parallel to the filament in which it resides. The average alignment angle of $\left\langle\theta_{\mathrm{HF}}\right\rangle=43.30^{\circ} \pm 0.12^{\circ}$ is smaller than for the SDSS galaxies. In addition, from $\mathrm{N}$-body simulation we select a sample of dark matter halos, with mass distribution equivalent to the host halos of central galaxies in SDSS galaxy catalog. We find the alignment signals for the dark matter halos are stonger than those of central galaxies in SDSS galaxy catalog, and the average alignment angle is $\left\langle\theta_{\mathrm{HF}}\right\rangle=42.19^{\circ} \pm 0.38^{\circ}$. This suggests a net misalignment between the major axes of galaxies and their dark matter halos, in excellent agreement with a number of previous alignment studies (e.g., Kang et al.|2007; Wang et al. 2008; Okumura et al. 2009; Agustsson \& Brainerd 2010; Li et al. 2013).

\subsubsection{Dependence on Galaxy Properties}

The large number $(212,046)$ of filament galaxies in our sample allows us to study how the galaxy-filament alignment signal depends on various galaxy properties. We start by using the galaxy group catalog to split the galaxy population into centrals (defined as the brightest group members) and satellites (those group members that are not centrals). These are further subdivided in 'red' and 'blue' according to their ${ }^{0.1}(g-r)$ colors: galaxies with ${ }^{0.1}(g-r) \geq 0.83$ are called 'red', while those with ${ }^{0.1}(g-r)<0.83$ are called 'blue'. The value 0.83 roughly corresponds to the bimodal scale in the color-magnitude relation (Weinmann et al. 2006).

Fig. 4 shows the galaxy shape-filament alignment signals for the resulting four sub-samples. Central galaxies reveal a remarkably strong color-dependence. While red centrals show strong alignment along their filaments, with $\left\langle\theta_{\mathrm{GF}}\right\rangle=43.26^{\circ} \pm 0.11^{\circ}$, virtually identical to that of the dark matter halos (cf. Fig. 3), blue filament centrals are only marginally aligned, with an average misalign-

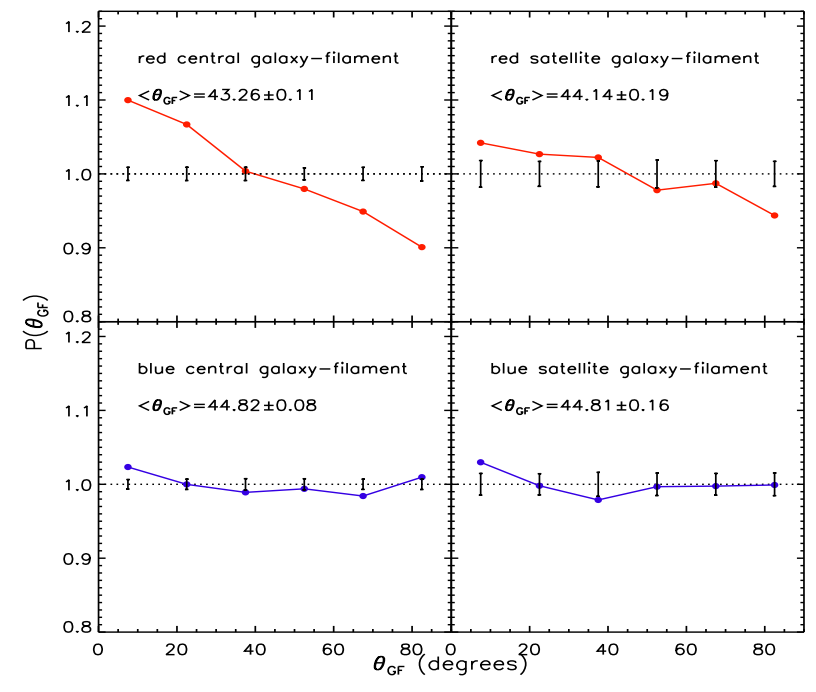

Fig. 4.- Same as Fig. 2 but for different subsamples ('red' and 'blue') of central and satellite galaxies, as indicated.

ment angle that is consistent with no alignment at the $2.3 \sigma$-level. Satellite galaxies, overall, are less strongly aligned with the filaments in which their host-groups reside than centrals. In particular, the orientations of blue satellites are consistent at the $1.2 \sigma$-level with having a random (projected) orientation with respect to their filament. In the case of red satellites, however, the alignment signal is $\left\langle\theta_{\mathrm{GF}}\right\rangle=44.14^{\circ} \pm 0.19^{\circ}$, which is significant at the $4.5 \sigma$-level.

A quantitatively similar dependence on galaxy color has been found for the alignment between the orientation of central galaxies and the angular distribution of their satellites (Yang et al. 2006; Azzaro et al. 2007; Wang et al. 2008; Agustsson \& Brainerd 2010), and suggests that red centrals are more accurately aligned with their host halo than blue centrals. We caution, though, that, as demonstrated by Kang et al. (2007), the interloper fraction (in the group catalog) is larger among blue centrals than among red centrals, which may cause a stronger dilution of the alignment signal for the blue centrals (since satellite galaxies show a weaker alignment signal). We will return to the interpretation of these findings in 4

Finally, we also investigate how the galaxy-filament alignment strength depends on the luminosity (stellar mass) of the galaxy, as well as on the mass of its dark matter host halo. To that extent we split the galaxy sample in equal sub-samples according to their $r$-band magnitude, and calculate $\left\langle\theta_{\mathrm{GF}}\right\rangle$ for each subsamples. Fig. 5 shows $\left\langle\theta_{\mathrm{GF}}\right\rangle$ versus ${ }^{0.1} M_{r}-5 \log h$. Results are shown separately for all color (left panels), red (middle panels) and blue galaxies (right panels), as well as separately for all (upper panels) and central (lower panels) galaxies. As we have the halo mass estimation for each group, we can obtain the average halo masses that correspond to given $r$-band magnitudes of central galaxies, which are indicated in the top axes of the lower raw panels. Overall, the alignment signal is stronger for brighter (massive) and redder galaxies, and for centrals in more massive halos. For blue galaxies the alignment is very weak at luminous end and almost absent at faint end. For red galaxies, 


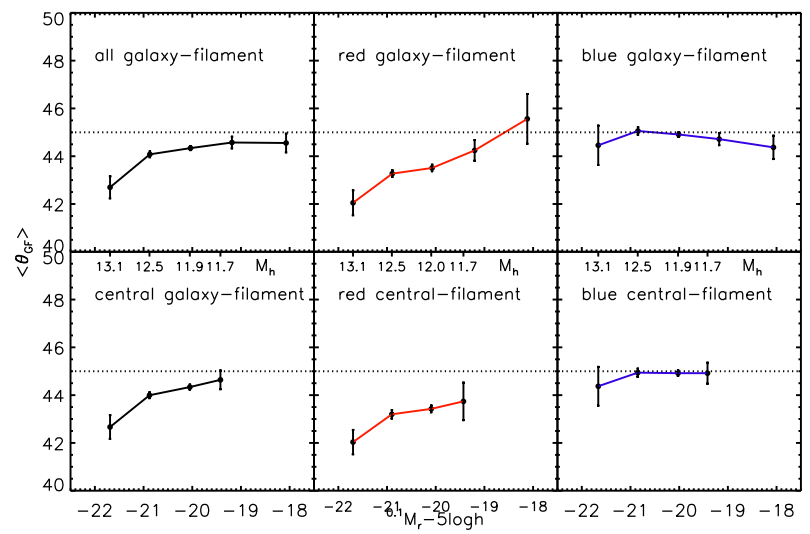

FIG. 5.- Average angle $\left\langle\theta_{\mathrm{GF}}\right\rangle$ as a function of the absolute magnitude ${ }^{0.1} M_{r}-5 \log h$ for filament galaxies. The average halo masses that correspond to given r-band magnitudes of central galaxies are indicated in the top axes of the lower panels.

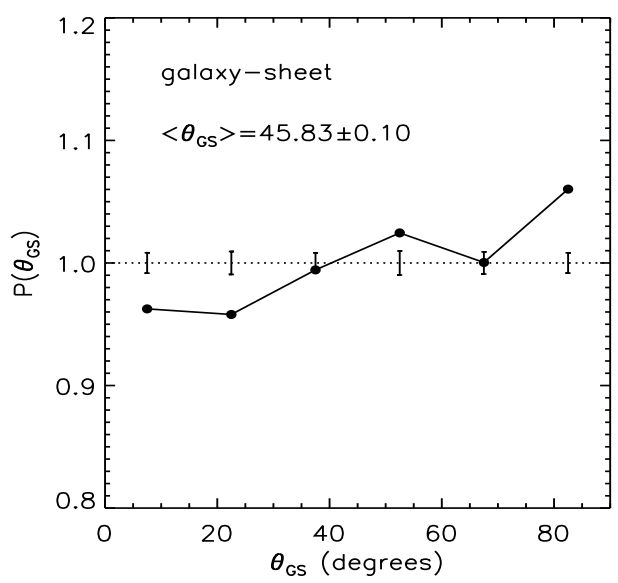

FIG. 6.- Normalized probability distribution of the angle $\theta_{\mathrm{GS}}$ between the projected orientation of the major axis of SDSS galaxies and that of the normal vector of the sheet in which its group resides. As before, the error bars indicate the scatter obtained from 100 realizations in which the orientations of the galaxies have been randomized.

significant alignment exist for all galaxies brighter than ${ }^{0.1} M_{r}-5 \log h=-20$, corresponding to a halo mass of $10^{12} h^{-1} \mathrm{M}_{\odot}$ for central galaxies.

A similar dependence of the halo-filament alignment on halo mass has been found in our N-body simulations. More massive halos in filaments have stronger alignment signals (Zhang et al. 2009). Here we perform the similar analysis for halos in difference mass ranges, we find $\left\langle\theta_{\mathrm{HF}}\right\rangle=42.18 \pm 0.61$ at $\log \left(M_{h}\right)=12.0$, $\left\langle\theta_{\mathrm{HF}}\right\rangle=41.25 \pm 1.05$ at $\log \left(M_{h}\right)=12.5$. Again the alignment signial is slightly (significantly) larger than that of red (blue) central galaxies in the same mass range.

\subsection{The Alignment between Galaxy Shape and Sheet}

Having discussed the alignment between galaxies and filaments, we now turn our attention to the galaxy-sheet alignment. To that extent we now focus on the 61,662 member galaxies of the 57,169 groups that reside in a sheet environment, and measure the probability distribution functions for their alignment angle $\theta_{\mathrm{GS}}$, between

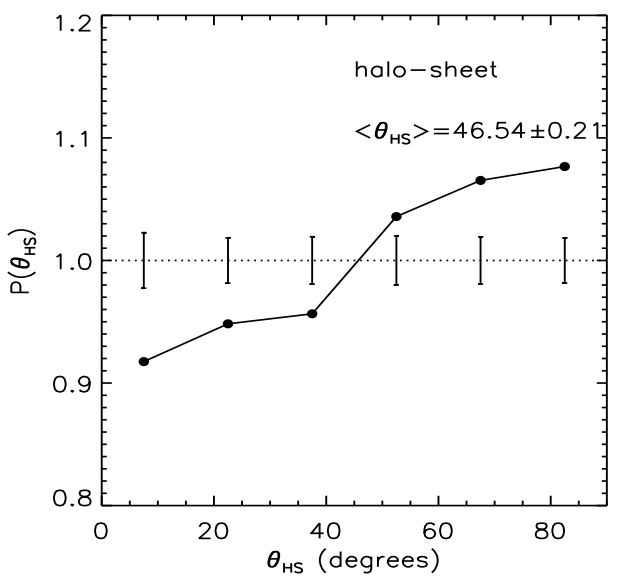

FIG. 7.- Same as Fig. [6 but for the alignment angle $\theta_{\text {HS }}$ between the projected orientation of a dark matter halo and that of the sheet in which it resides. These results have been obtained using the numerical $N$-body simulation described in 2.4

the projected orientations of the galaxy and the normal vector of the sheet.

The results for all sheet galaxies are shown in Fig. 6. which indicate a significant anti-alignment between galaxy major axis and sheet-normal of $\left\langle\theta_{\mathrm{GS}}\right\rangle=$ $45.83^{\circ} \pm 0.10^{\circ}$. This indicates that sheet galaxies have their minor axes preferentially aligned along the normal vector of the sheet. Fig. 7 shows the alignment signal between the projected orientation of dark matter halos and the normal vector of their sheets, as obtained from the $N$-body simulation discussed in 2.4. Similar to the SDSS data, their is a significant anti-alignment. Although the mean misalignment angle $\left(46.54^{\circ}\right)$ is somewhat larger than that for the galaxies, this difference is, given the larger errorbar for the dark matter halos, not significant. These results regarding the dark matter halos are in qualitative agreement with a number of previous studies based on numerical simulations in $\Lambda \mathrm{CDM}$ cosmologies (e.g., Aragón-Calvo et al. 2007; Hahn et al. 2007; Zhang et al. 2009).

\subsubsection{Dependence on Galaxy Properties}

In the following, we investigate the dependence of the galaxy-sheet alignment on the galaxy properties. Similar to what we did for the galaxy-filament alignments, we first split the population in centrals and satellites, which are then further subdivided in 'red' and 'blue', based on their ${ }^{0.1}(g-r)$ color. As shown in Fig. 8, the color dependence of the shape-sheet alignment is similar to that of the shape-filament alignment. Red galaxies are more strongly aligned with the plane of the sheet than blue galaxies, and the alignment for centrals is much stronger than for satellites. In fact, neither red nor blue satellites show a significant (more than $2 \sigma$ ) alignment signal. The strongest alignment signal is found for red centrals, for which $\left\langle\theta_{\mathrm{GS}}\right\rangle=46.75^{\circ} \pm 0.19^{\circ}$ (a $9.2 \sigma$ significant detection of alignment). Interestingly, the strength of this alignment signal is again very similar to that of the dark matter halos in the simulation (cf. Fig. 7), indicating once more that red centrals may be strongly aligned with their host halos.

Finally, Fig. 9] shows the dependence of $\left\langle\theta_{\mathrm{GS}}\right\rangle$ on galaxy 


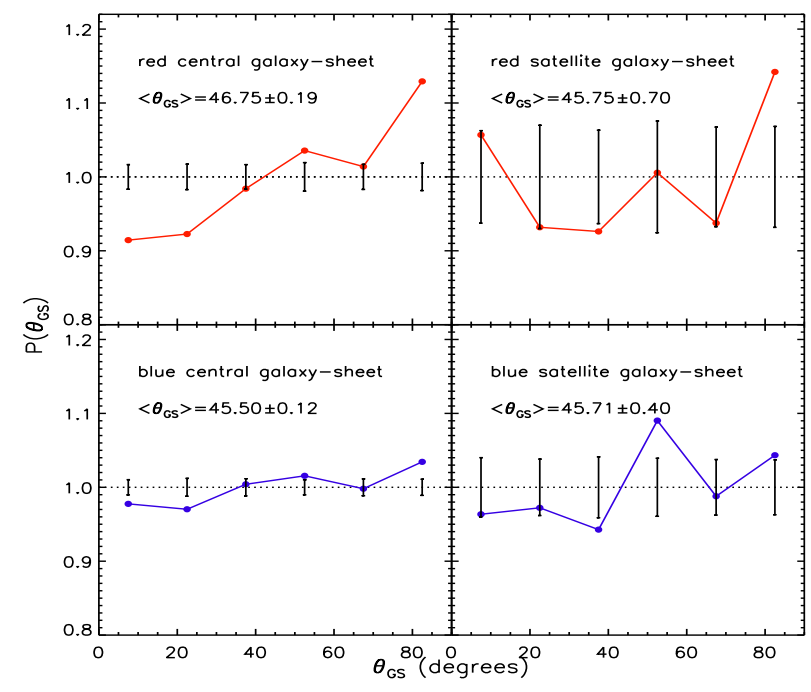

FIG. 8. - Same as Fig. 4 but for the galaxy-sheet alignment angle, $\theta_{\mathrm{GS}}$.

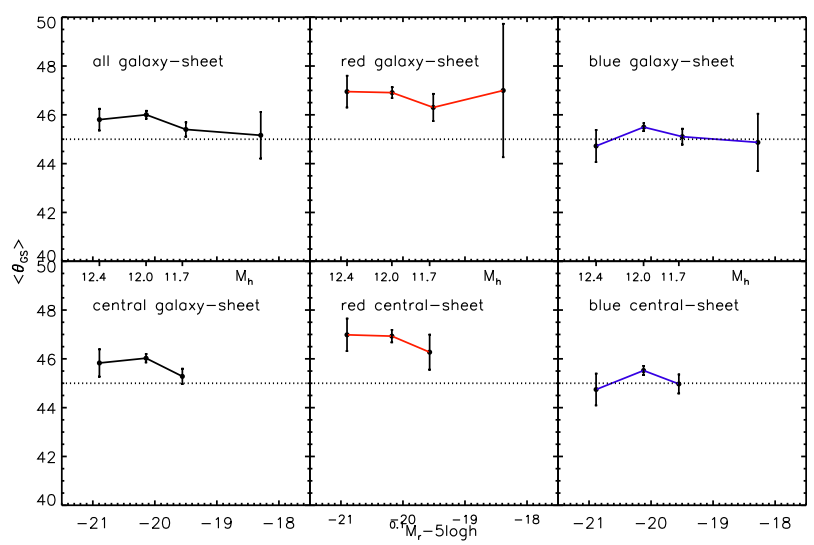

FIG. 9. - Same as Fig. 5 but for the galaxy-sheet alignment angle, $\left\langle\theta_{\mathrm{GS}}\right\rangle$.

luminosity for subsamples divided according to color, and according to central versus total. In agreement with the results for the galaxy-filament alignment, the alignment is typically stronger and significant only for red galaxies. Within a given color subsample, the dependence on galaxy luminosity is rather weak.

\subsection{The Alignment between Galaxy Shape and Tidal Tensor}

In the previous subsections, we investigated the alignments of filament and sheet galaxies with their respective cosmic web structure. This analysis, however, ignored the cluster and void galaxies, which combined make up about $30 \%$ of the galaxy population in our sample. In this subsection, we test the alignment of the entire galaxy population $(395,841$ galaxies in cluster, sheet, filament, and void environments) with the local tidal field. As discussed in 2.2 the latter has been calculated from the SDSS DR7 using the distribution of galaxy groups with assigned halo masses $M_{\mathrm{h}} \geq 10^{12} h^{-1} \mathrm{M}_{\odot}$. At the center of mass of each group we calculate the eigenvalues $\lambda_{1}, \lambda_{2}$ and $\lambda_{3}\left(\lambda_{1}>\lambda_{2}>\lambda_{3}\right)$ of the tidal tensor.
In what follows we refer to the corresponding eigenvectors as $\boldsymbol{t}_{1}, \boldsymbol{t}_{2}$, and $\boldsymbol{t}_{3}$, respectively. The projected orientation of $\boldsymbol{t}_{1}, \boldsymbol{t}_{2}$, and $\boldsymbol{t}_{3}$, at the location $\boldsymbol{x}$ of a group, is computed using the equation (4), where $\Delta \boldsymbol{x}$ is the 3D eigenvector corresponding to the eigenvalue $\lambda_{1}, \lambda_{2}$ and $\lambda_{3}$, respectively.

The first row of Fig. 10 shows the alignment between the projected orientations of the three eigenvectors of the tidal field and that of the major axis of the galaxies. For galaxies in sheet environments, $\boldsymbol{t}_{1}$ indicates the direction perpendicular to the sheet-plane, and the alignment with $\boldsymbol{t}_{1}$ is therefore identical to $\theta_{\mathrm{GS}}$. Similarly, the the alignment with $\boldsymbol{t}_{3}$ is identical to $\theta_{\mathrm{GF}}$ for filament galaxies. Here, though, we include all galaxies, independent of their environment, and we show the alignment angles with all three eigenvectors of the tidal tensor. There is a very significant alignment $(14 \sigma)$ with the eigenvector associated with the largest eigenvalue, $\boldsymbol{t}_{1}$, which is expected from the fact that we had already seen ( 93.2 ) that sheet galaxies are preferentially oriented perpendicular to the normal vector of the sheet. There is no indication of any alignment between galaxies and the intermediate eigenvector, $\boldsymbol{t}_{2}$, but again a very significant alignment $(13 \sigma)$ with the eigenvector associated with the smallest eigenvalue, $\boldsymbol{t}_{3}$. The latter is again expected from the fact that filament galaxies (which make up $53.6 \%$ of the entire galaxy population) are aligned with the direction of their filaments.

The second row in Fig 10 shows the alignments between the projected orientations of dark matter halos and the eigenvectors of the tidal tensor (also in projection), obtained from the $N$-body simulation. Halos are anti-aligned with $\boldsymbol{t}_{1}$, positively aligned with $\boldsymbol{t}_{3}$, and not aligned with $\boldsymbol{t}_{2}$, in excellent agreement with previous studies (e.g., Wang et al. 2011; Libeskind et al. 2013; Tempel et al. 2013a). These results are also in good, qualitative agreement with the alignments seen for the SDSS galaxies, although the alignment for halos is found to be significantly stronger than that for the entire galaxy population. As shown above, red central galaxies seem to be far more strongly aligned with their sheets or filaments than blue centrals or satellites. The third row of Fig 10 therefore shows the alignments of red centrals with the eigenvectors of the tidal tensor. These are found to be amazingly similar to those of the dark matter halos, supporting the notion that red centrals may be strongly aligned with their host halos. In contrast, blue centrals do not show any significant alignments with the eigenvectors of the tidal tensor (the fourth row of the figure).

\section{SUMMARY AND DISCUSSION}

Using galaxy groups selected from the SDSS DR7, we have examined the alignment between the major axes of galaxies and their surrounding large-scale structure, especially the filaments and sheets. We characterized the large scale structure using the tidal tensor, which we reconstructed using galaxy groups with an assigned halo mass $M_{\mathrm{h}} \gtrsim 10^{12} h^{-1} \mathrm{M}_{\odot}$. Based on the eigenvalues of the tidal tensor at the location of each galaxy group, we have split the galaxy population into four environments: cluster, filament, sheet and void.

We have shown that the major axes of galaxies in a filament environment are preferentially aligned along the direction of the filament (defined as the direc- 


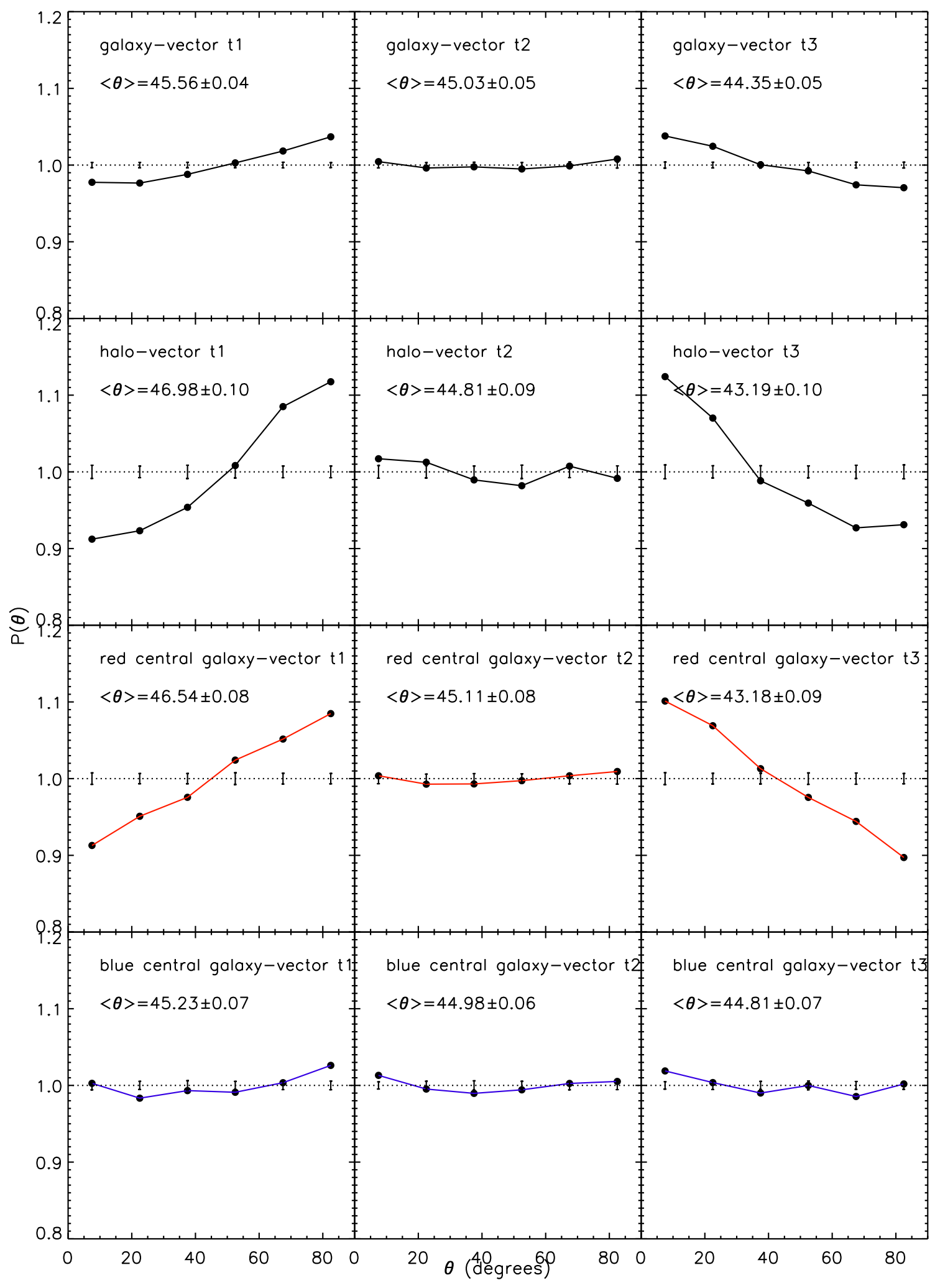

FIG. 10.- Probability distribution of the angle $\theta$ between the orientation of the (projected) major axis of the galaxy and the eigenvectors $\boldsymbol{t}_{1}$ (left column), $\boldsymbol{t}_{2}$ (middle column), and $\boldsymbol{t}_{3}$ (right column) of the tidal tensor field for all galaxies in the SDSS (first row), for dark matter halos in our $N$-body simulation (second row), for red central galaxies in the SDSS (third row), and for blue central galaxies in the SDSS (fourth row). The eigenvector $\boldsymbol{t}_{i}$ corresponds to the eigenvalue $\lambda_{i}$, where $\lambda_{1} \geq \lambda_{2} \geq \lambda_{3}$. As before, errorbars indicate the scatter obtained from 100 realizations in which the orientations of the galaxies or dark matter halos have been randomized. 
tion of the eigenvector of the tidal tensor associated with the single negative eigenvalue), while galaxies in a sheet environment are preferentially aligned perpendicular to the normal vector of the sheet (defined as the direction of the eigenvector of the tidal tensor associated with the single positive eigenvalue). In both cases, the alignment is strongest for red central galaxies, is weak for blue centrals, and is virtually absent for satellite galaxies7. In addition, the alignment strength is found to increase with galaxy luminosity and/or stellar mass.

We also computed the alignments between the orientations of all galaxies (independent of their environment) and the three eigenvectors of the tidal tensor at the location of the galaxy group in which they reside. Consistent with the above results, we find a strong antialignment (at $14 \sigma$ significance) with the eigenvector $\boldsymbol{t}_{1}$ associated with the largest eigenvalue, no alignment at all with the eigenvector $\boldsymbol{t}_{2}$ associated with the intermediate eigenvalue, and strong, positive alignment (at $13 \sigma$ significance) with the eigenvector $\boldsymbol{t}_{3}$ assocated with the smallest eigenvalue. In the case of red, central galaxies, the significance of these alignments becomes even stronger: $19.3 \sigma$ and $20.2 \sigma$ in the cases of the alignments with $\boldsymbol{t}_{1}$ and $\boldsymbol{t}_{3}$, respectively. In contrast, for blue galaxies the alignments are always quite weak.

Using a large, $N$-body simulation, we also calculated how the shapes of dark matter halos are aligned with their filaments and sheets (in projection). Our results are in excellent agreement with previous studies (e.g., Hahn et al. 2007; Zhang et al. 2009; Wang et al. 2011; Libeskind et al. 2013; Tempel et al. 2013a). Interestingly, we find alignment strengths for the dark matter halos that are indistinguishable from those of red centrals, strongly suggesting that red central galaxies are strongly aligned with their dark matter host halos. This is consistent with the results obtained in Yang et al. (2006); Kang et al. (2007); Wang et al. (2008); Okumura et al. (2009), all of which show that red centrals and their host halos are more strongly aligned than the halo - filament and halo - sheet alignments found here. These measured alignments between galaxy shape and the surrounding large-scale structure from observation may indicate that galaxy formation is affected by large-scale environments. The alignment signals may be a natural result of how galaxies accrete material via streams along the direction of the filaments, and the thin plane of the sheets. They also suggest that the shapes and orientations of red central galaxies are significantly affected by such accretion.

The fact that blue centrals show an alignment signal that is much weaker than that of dark matter halos implies that blue central galaxies are only poorly aligned with the shape of their host halos. These results are in qualitative agreement with previous studies (e.g., Yang et al. 2006; Azzaro et al. 2007; Wang et al. 2008; Faltenbacher et al. 2009; Okumura et al. 2009; Agustsson \& Brainerd 2010; Li et al. 2013), most of which focussed on the alignment of central galaxies with the spatial (angular) distribution of satellite galaxies.
The fact that the projected orientation of blue centrals is positively aligned with that of the filaments (albeit at a marginaly level), is also interesting in light of some recent results by Tempel et al. (2013b) and Tempel \& Libeskind (2013), who found evidence that the rotation axes of disk galaxies are (weakly) aligned with the directions of their filaments. A similar result was obtained by Hahn et al. (2010), albeit for massive disk galaxies in hydrodynamical simulations. Using $N$-body simulations, Wang et al. (2011) found that the spin axis of a dark matter halo is correlated with the directions of the tidal directions, but the correlation is significantly halo mass dependent. For halos with masses as low as $\sim 10^{12} h^{-1} \mathrm{M}_{\odot}$, the correlation becomes rather weak but is still significant. In as far as 'blue' galaxies are 'disk' galaxies with spin axis similar to their host halos, our results are not consistent with these results. We caution, though, that all three studies performed their analysis in $3 \mathrm{D}$, while our analysis is done in projection in $2 \mathrm{D}$. In addition, all studies only found a positive alignment between spin vector and filament direction for massive disk galaxies and halos. It might well be that projection effects, and the inclusion of large numbers of less massive galaxies, largely erase the signatures of the mild alignment effects seen in Hahn et al. (2010), Wang et al. (2011), Tempel et al. (2013b), and Tempel \& Libeskind (2013).

If galaxies are strongly aligned with sheets and filaments, as we find here at least for red central galaxies, then galaxies should also be mutually aligned over scales of those sheets and filaments. Such alignments have important ramifications for cosmic shear measurements based on the orientation correlation of source galaxies (e.g. Okumura et al. 2009; Kirk et al. 2012; Joachimi et al. 2013). Our results can help in modeling such contaminations. In particular, our finding that the alignments are strong only for red bright galaxies suggests that the contaminations can be reduced by using only blue and relatively faint sources.

\section{ACKNOWLEDGEMENTS}

This work is supported by the national science foundation of China (grant Nos. 11203054, 10925314, 11128306, 11121062, 11233005, 11073017), NCET-110879, the CAS/SAFEA International Partnership Program for Creative Research Teams (KJCX2-YW-T23), and the Shanghai Committee of Science and Technology, China (grant No. 12ZR1452800). HJM would like to acknowledge the support of NSF AST-0908334 and NSF AST-1109354.

Funding for the SDSS and SDSS-II was provided by the Alfred P. Sloan Foundation, the Participating Institutions, the National Science Foundation, the U.S. Department of Energy, the National Aeronautics and Space Administration, the Japanese Monbukagakusho, the Max Planck Society, and the Higher Education Funding Council for England. The SDSS was managed by the Astrophysical Research Consortium for the Participating Institutions.

\section{REFERENCES}


Abazajian, K. N., Adelman-McCarthy, J. K., Agüeros, M. A., et al. 2009, ApJS, 182, 543

Agustsson, I., \& Brainerd, T. G. 2010, ApJ, 709, 1321

Altay, G., Colberg, J. M., \& Croft, R. A. C. 2006, MNRAS, 370, 1422

Aragón-Calvo, M. A., van de Weygaert, R., Jones, B. J. T., \& van der Hulst, J. M. 2007, ApJ, 655, L5

Aragon-Calvo, M. A. 2012, preprint (arXiv:1210.7871)

Azzaro, M., Patiri, S. G., Prada, F., \& Zentner, A. R. 2007, MNRAS, 376, L43

Basilakos, S., Plionis, M., Yepes, G., Gottlöber, S., \& Turchaninov, V. 2006, MNRAS, 365, 539

Berta, Z. K., Jimenez, R., Heavens, A. F., \& Panter, B. 2008, MNRAS, 391, 197

Bett, P., Eke, V., Frenk, C. S., et al. 2007, MNRAS, 376, 215

Blanton M. R. et al., 2003, AJ, 125, 2348

Blanton, M. R. et al., 2005, AJ, 129, 2562

Bond J. R., Kofman L., Pogosyan D., 1996, Nature, 380, 603

Brainerd, T. G. 2005, ApJ, 628, L101

Brunino, R., Trujillo, I., Pearce, F. R., \& Thomas, P. A. 2007, MNRAS, 375, 184

Byrd, G., \& Valtonen, M. 1990, ApJ, 350, 89

Choi, Y., Park, C., \& Vogeley, M. S. 2007, ApJ, 658, 884

Choi, Y.-Y., Park, C., Kim, J., et al. 2010, ApJS, 190, 181

Codis, S., Pichon, C., Devriendt, J., et al. 2012, MNRAS, 427, 3320

Colberg, J. M., Krughoff, K. S., \& Connolly, A. J. 2005, MNRAS, 359,272

Cuesta, A. J., Betancort-Rijo, J. E., Gottlöber, S., et al. 2008, MNRAS, 385, 867

Dalal, N., White, M., Bond, J. R., \& Shirokov, A. 2008, ApJ, 687, 12

Davis, M., Efstathiou, G., Frenk, C. S., \& White, S. D. M. 1985 ApJ, 292, 371

Debattista, V. P., Roškar, R., Valluri, M., et al. 2013, MNRAS, 434, 2971

Faltenbacher, A., Gottlöber, S., Kerscher, M., Müller, V. 2002 A\&A, 395, 1

Faltenbacher, A., Li, C., Mao, S., et al. 2007, ApJ, 662, L71

Faltenbacher, A., Li, C., White, S. D. M., et al. 2009, Research in Astronomy and Astrophysics, 9, 41

Gao, L., Springel, V., \& White, S. D. M. 2005, MNRAS, 363, L66 Godłowski, W., Piwowarska, P., Panko, E., \& Flin, P. 2010, ApJ, 723,985

Godłowski, W. 2011, International Journal of Modern Physics D, 20,1643

Godłowski, W. 2012, ApJ, 747, 7

Gottlöber, S., Klypin, A., \& Kravtsov, A. V. 2001, ApJ, 546, 223

Hahn, O., Porciani, C., Carollo, C. M., \& Dekel, A. 2007a, MNRAS, 375, 489

Hahn, O., Carollo, C. M., Porciani, C., \& Dekel, A. 2007b, MNRAS, 381, 41

Hahn, O., Porciani, C., Dekel, A., \& Carollo, C. M. 2009 MNRAS, 398, 1742

Hahn, O., Teyssier, R., \& Carollo, C. M. 2010, MNRAS, 405, 274

Hao, J., Kubo, J. M., Feldmann, R., et al. 2011, ApJ, 740, 39

Jimenez, R., Slosar, A., Verde, L., et al. 2010, MNRAS, 404, 975

Jing, Y. P., Suto, Y., \& Mo, H. J. 2007, ApJ, 657, 664

Joachimi B., Semboloni E., Hilbert, S., Bett, P. E., Hartlap, J., Hoekstra, H., Schneider, P. 2013, arXiv1305.5791

Kang, X., van den Bosch, F. C., Yang, X., et al. 2007, MNRAS, 378,1531

Kasun, S. F., \& Evrard, A. E. 2005, ApJ, 629, 781

Kirk, D., Rassat, A., Host, O., Bridle, S. 2012, MNRAS, 424, 1647

Lacey, C., \& Cole, S. 1993, MNRAS, 262, 627

Li, C., Jing, Y. P., Faltenbacher, A., \& Wang, J. 2013, ApJ, 770, L12
Li, Z., Wang, Y., Yang, X., et al. 2013, ApJ, 768, 20

Libeskind, N. I., Hoffman, Y., Forero-Romero, J., et al. 2013, MNRAS, 428, 2489

Mo, H. J., van den Bosch, F. C., \& White, S. D. M. 2010, Galaxy Formation and Evolution (Cambridge: Cambridge University Press)

Muldrew, S. I., Croton, D. J., Skibba, R. A., et al. 2012, MNRAS, 419,2670

Nierenberg, A. M., Auger, M. W., Treu, T., Marshall, P. J., \&

Fassnacht, C. D. 2011, ApJ, 731, 44

Okumura, T., Jing, Y. P., \& Li, C. 2009, ApJ, 694, 214

Park, C., \& Choi, Y. 2005, ApJ, 635, L29

Patiri, S. G., Cuesta, A. J., Prada, F., Betancort-Rijo, J., \& Klypin, A. 2006, ApJ, 652, L75

Paz, D. J., Stasyszyn, F., \& Padilla, N. D. 2008, MNRAS, 389 1127

Paz, D. J., Sgró, M. A., Merchán, M., \& Padilla, N. 2011, MNRAS, 414, 2029

Pereira, M. J., \& Kuhn, J. R. 2005, ApJ, 627, L21

Sales L., \& Lambas D. G. 2004, MNRAS, 348, 1236

Schneider, M. D., Cole, S., Frenk, C. S., et al. 2013, MNRAS, 1666

Sheth, R. K., \& Tormen, G. 2004, MNRAS, 350, 1385

Skielboe, A., Wojtak, R., Pedersen, K., Rozo, E., \& Rykoff, E. S. 2012, ApJ, 758, L16

Springel, V. 2005, MNRAS, 364, 1105

Tempel, E., Libeskind, N. I., Hoffman, Y., Liivamägi, L. J.,\& Tamm, A. 2013a, preprint (arXiv:1307.1232)

Tempel, E., Stoica, R. S., \& Saar, E. 2013b, MNRAS, 428, 1827

Tempel, E., \& Libeskind, N. I. 2013, preprint (arXiv:1308.2816)

Trowland, H. E., Lewis, G. F., \& Bland-Hawthorn, J. 2013, ApJ, 762,72

Wang, H. Y., Mo, H. J., \& Jing, Y. P. 2007, MNRAS, 375, 633

Wang, H., Mo, H. J., Jing, Y. P., Guo, Y., van den Bosch, F. C. \& Yang, X. 2009, mnras, 394, 398

Wang, H., Mo, H. J., Jing, Y. P., Yang, X., \& Wang, Y. 2011, MNRAS, 413, 1973

Wang, H., Mo, H. J., Yang, X., \& van den Bosch, F. C. 2012, MNRAS, 420, 1809

Wang, Y., Yang, X., Mo, H. J., et al. 2008, MNRAS, 385, 1511

Wang, Y., Park, C., Yang, X., Choi, Y.-Y., \& Chen, X. 2009 ApJ, 703, 951

Wang, Y., Park, C., Hwang, H. S., \& Chen, X. 2010, ApJ, 718, 762

Weinmann, S. M., van den Bosch, F. C., Yang, X., \& Mo, H. J. 2006, MNRAS, 366, 2

White, S. D. M. 1984, ApJ, 286, 38

White S. D. M. 1996, in Cosmology and Large-Scale Structure, edited by R. Schaefer. J. Silk. M. Spiro, J. Zinn-Justin, Dordrecht: Elsevier, astro-ph/9410043

Yang, X., Mo, H. J., van den Bosch, F. C., \& Jing, Y. P. 2005 MNRAS, 356, 1293

Yang, X., van den Bosch, F. C., Mo, H. J., et al. 2006, MNRAS, 369, 1293

Yang, X., Mo, H. J., van den Bosch, F. C., et al. 2007, ApJ, 671 153

York, D. G., Adelman, J., Anderson, J. E., Jr., et al. 2000, AJ 120,1579

Zel'dovich, Y. B. 1970, A\&A, 5, 84

Zhang, Y., Yang, X., Faltenbacher, A., et al. 2009, ApJ, 706, 747

Zhao, D. H., Mo, H. J., Jing, Y. P., Börner, G. 2003, MNRAS, 339,12

Zhao, D. H., Jing, Y. P., Mo, H. J., Börner, G. 2009, ApJ, 707, 354 